\title{
Article
}

Subscriber access provided by UNIV OF NEW ENGLAND ARMIDALE

\section{Core/shell Nanoparticles of Non-Stoichiometric Zn-Mn and Zn-Co Ferrites as Thermosensitive Heat Sources for Magnetic Fluid Hyperthermia} Vanessa Pilati, Rafael Cabreira Gomes, Guilherme Siqueira Gomide, Priscilla Coppola, Franciscarlos Gomes Silva, Fabio Luis Oliveira Paula, Régine Perzynski, Gerardo F. Goya, Renata Aquino, and Jérôme Depeyrot

J. Phys. Chem. C, Just Accepted Manuscript • DOI: 10.1021/acs.jpcc.7b11014 • Publication Date (Web): 22 Jan 2018

Downloaded from http://pubs.acs.org on January 23, 2018

\section{Just Accepted}

"Just Accepted" manuscripts have been peer-reviewed and accepted for publication. They are posted online prior to technical editing, formatting for publication and author proofing. The American Chemical Society provides "Just Accepted" as a free service to the research community to expedite the dissemination of scientific material as soon as possible after acceptance. "Just Accepted" manuscripts appear in full in PDF format accompanied by an HTML abstract. "Just Accepted" manuscripts have been fully peer reviewed, but should not be considered the official version of record. They are accessible to all readers and citable by the Digital Object Identifier (DOI®). "Just Accepted" is an optional service offered to authors. Therefore, the "Just Accepted" Web site may not include all articles that will be published in the journal. After a manuscript is technically edited and formatted, it will be removed from the "Just Accepted" Web site and published as an ASAP article. Note that technical editing may introduce minor changes to the manuscript text and/or graphics which could affect content, and all legal disclaimers and ethical guidelines that apply to the journal pertain. ACS cannot be held responsible for errors or consequences arising from the use of information contained in these "Just Accepted" manuscripts. 


\title{
Core/Shell Nanoparticles of Non-Stoichiometric Zn-Mn and Zn-Co Ferrites as Thermosensitive Heat Sources for Magnetic Fluid Hyper-
}

\section{thermia}

\author{
Vanessa Pilati, ${ }^{\dagger}$ Rafael Cabreira Gomes,,,$+ \S$ Guilherme Gomide, ${ }^{\dagger}$ Priscilla Coppola, $\perp, \#$ Franciscarlos \\ G. Silva, ${ }^{\dagger, \perp}$ Fábio. L. O. Paula, ${ }^{\dagger}$ Régine Perzynski,,$\$$ Gerardo F. Goya, ${ }^{\ddagger}$ Renata Aquino, ${ }^{\dagger, \perp}$ and Jérôme \\ Depeyrot*, ${ }^{*}$.
}

\author{
†3NANO Group, Instituto de Física, Universidade de Brasília, UnB, 70919-970 Brasília - DF, Brazil. \\ ‡Instituto de Nanociencia de Aragon (INA), University of Zaragoza, 50018 Zaragoza, Spain. \\ §Sorbonne Universités, UPMC Univ. Paris 06, CNRS, Lab. PHENIX, 4 Place Jussieu, 75005 Paris, France. \\ $\perp$ 3NANO Group, Laboratório de Nanociência Ambiental e Aplicada, Universidade de Brasília, Faculdade UnB \\ Planaltina (FUP-UnB), 73345-010 Brasília - DF, Brazil. \\ \#3NANO Group, Instituto de Química, Universidade de Brasília, UnB, 70919-970 Brasília - DF, Brazil.
}

\begin{abstract}
We report on the suitability of core/shell nanoparticles (NPs) for magnetic fluid hyperthermia in a selfregulated and theranostic approach. Aqueous magnetic colloids based on core/shell $\mathrm{Zn}_{\mathrm{x}} \mathrm{Mn}_{\mathrm{y}} \mathrm{Fe}_{\mathrm{z}} \mathrm{O}_{4} @ \gamma-\mathrm{Fe}_{2} \mathrm{O}_{3}$ and $\mathrm{Zn}_{\mathrm{x}} \mathrm{Co}_{\mathrm{y}} \mathrm{Fe}$ ${ }_{z} \mathrm{O}_{4} @ \gamma-\mathrm{Fe}_{2} \mathrm{O}_{3}$ NPs were produced by a three-step chemical synthesis. Systematic deviations from stoichiometry was observed with increasing Zn substitution for both series of samples. We investigated how the chemical composition affects saturation magnetization, magnetic anisotropy and thermomagnetic properties of these core/shell NPs. The heating efficiency through specific power absorption (SPA) was analyzed in the framework of the linear response theory. SPA values obtained for NPs presenting different contrast of anisotropy between the core and shell materials indicate no evidence of enhanced exchange coupling contribution to the heating efficiency.
\end{abstract}

\section{INTRODUCTION}

Hyperthermia is a powerful and well-known oncological therapy, aiming to heat cancer cells up to $41-45^{\circ} \mathrm{C}$ to trigger cytotoxic effects with minimum harm on the surrounding tissues. Local heating of tumorous cells can be achieved by using magnetic NPs as heat sources in magnetic fluid hyperthermia (MFH) given their ability to convert magnetic energy into heat when submitted to a timevarying magnetic field ${ }^{1-3}$. In addition, since magnetic NPs provide contrast enhancement in magnetic resonance imaging (MRI), they are being explored as dual-purpose systems for therapy (MFH) and diagnostics (MRI) in a theranostic approach ${ }^{4}$.

The heat produced by ferrimagnetic single-domain NPs under a time-varying magnetic field is related to dynamic hysteresis losses produced by the relaxation of the magnetic moment 5,6 . The phenomenology of MFH arises from two mechanisms associated to the relaxation of the NPs' magnetic moment. When dispersed in a liquid carrier, an isolated magnetic NP can rotate and the associated Brownian relaxation time is $\tau_{B}=3 \eta V_{H} / k_{B} T, \eta$ being the viscosity of the medium, $V_{H}$ the NP hydrodynamic volume and $k_{B} T$ the thermal energy, with $k_{B}$ being the Boltzmann constant. The magnetic moment would also relax due to thermally induced fluctuations by overcoming an energy barrier of magnetic anisotropy $E_{a}$, a phenomenon well characterized by a Néel relaxation time ${ }^{7} \tau_{N}=\tau_{0} \exp \left(E_{a} / k_{B} T\right), \tau_{0}$ being the spin relaxation time of the order of $10^{-9} \mathrm{~s}$ (for a detailed review of Néel relaxation time expression see for example Ref. ${ }^{8}$ ). Assuming these two mechanisms are independent, the effective relaxation time is given by $\tau^{-1}=\tau_{N}^{-1}+\tau_{B}^{-1}$ and the dominant mechanism for the heating generation will be the faster one.

The power absorbed by the NPs is commonly described by the linear response theory (LRT) when the applied field $H_{0}$ is much smaller than the anisotropy field $H_{a}$ of the NPs and the dynamic magnetization of the system varies linearly with $H_{0} 5,6,9,10$. Within this approximation, the heating rates of the NPs present an optimum particle size, whose values depend strongly on the magnetic anisotropy ${ }^{5,6}$. The heating efficiency has been experimentally investigated as a function of several parameters, such as the particle size ${ }^{11-17}$, saturation magnetization, morphology $y^{14,18-22}$ and 
magnetic anisotropy11,14,23-25. Moreover, the efficiency of NPs with varying anisotropies and diameters in medium of different viscosities has been explored $11,12,17,23,26$. More recently, the role of magnetic dipolar interaction ${ }^{27-33}$ and NPs aggregation $18,21,27,28,34-38$ in power loss efficiency have emerged as a controversial issue and further studies are needed to better understand it.

Since the heating efficiency in MFH is strongly dependent on NPs individual properties like size, magnetization and anisotropy, a combination of different materials in a unique core/shell NP would be an interesting strategy to improve the $\mathrm{MFH}$ performance ${ }^{39-43}$. In the last years, few studies pointed out that the design of core/shell nanoparticles with a high contrast of anisotropy between shell and core provides a remarkable enhancement of the power loss efficiency ${ }^{19,44,45}$. It has been proposed that this observed increase of SPA would be related to the interfacial exchange coupling between magnetically soft and hard phases. Very recently ${ }^{46,47}$, the power absorption of such systems has been calculated by using a theoretical approach based on the LRT and considering the influence of both core and shell contributions as well as the exchange coupling at the interface. It is found that increasing the interface exchange constant would be an interesting strategy to achieve better efficiency with small core/shell soft/hard NPs.

We propose here to investigate the heating performance of core/shell NPs based on a hard/soft and soft/soft magnetic ferrite phases. Indeed, in a previous work ${ }^{48}$, an exchange bias field in ultrasmall $(\sim 3 \mathrm{~nm})$ core/shell $\mathrm{MnFe}_{2} \mathrm{O}_{4} @ \gamma-\mathrm{Fe}_{2} \mathrm{O}_{3}$ and $\mathrm{CoFe}_{2} \mathrm{O}_{4} @ \gamma-\mathrm{Fe}_{2} \mathrm{O}_{3}$ NPs has been measured. It is found that the magnitude of this exchange coupling field is larger for core/shell NPs based on harder ferrite core. Following reference ${ }^{44}$, this result would indicate that they could be more efficient for the conversion of electromagnetic energy into heat. In this paper, we also choose to modulate the thermomagnetic response by doping the NPs core with diamagnetic $\mathrm{Zn}^{2+}$ ions, since larger zinc content provides the weakening of the superexchange interactions. It therefore leads to increasing thermomagnetic coefficient ${ }^{49,50}\left(k_{T M}=-\Delta \sigma / \Delta T\right.$, where $\sigma$ is the magnetization per unit mass) and decreasing Curie temperature hence limiting the upper temperature reached by MFH to the safety range $\left(41-45^{\circ} \mathrm{C}\right){ }^{49-54}$. This characteristic would be very promising because it could enable a local self-regulated temperature control ${ }^{55,56}$, which would avoid damages to healthy tissues due to overheating.

In such a context, this paper reports on MFH performances of two series of electric double layered magnetic fluids (EDL-MF) based on core/shell $\mathrm{Zn}_{\mathrm{x}} \mathrm{Mn}_{\mathrm{y}} \mathrm{Fe}_{\mathrm{z}} \mathrm{O}_{4} @ \gamma-\mathrm{Fe}_{2} \mathrm{O}_{3}$ and $\mathrm{Zn}_{\mathrm{x}} \mathrm{Co}_{\mathrm{y}} \mathrm{Fe}_{\mathrm{z}} \mathrm{O}_{4} @ \gamma-\mathrm{Fe}_{2} \mathrm{O}_{3}$ NPs, with different $\mathrm{Zn} / \mathrm{Mn}$ and $\mathrm{Zn} / \mathrm{Co}$ proportions. These colloidal NPs are dispersed in aqueous medium by using a well-controlled synthesis route ${ }^{57-59}$ and present a long term thermodynamic stability thanks to the introduction of adjustable Coulomb's repulsive forces between them ${ }^{60,61}$. Intrinsic magnetic properties like magnetization and coercive field are explored in detail and allow to correlate them with the heating efficiency. The strong anisotropic character of Co cations when compared to $\mathrm{Zn}$ and $\mathrm{Mn}$ ones allows to tune the magnetic anisotropy constant and the contrast of anisotropy between the core and shell materials. Finally, we discuss these results in light of interfacial exchange coupling between core and shell phases.

\section{MATERIALS AND METHODS}

A. Synthesis of Nanoparticles and Magnetic Colloids

Magnetic colloids are synthesized in three steps, as described in more detail elsewhere ${ }^{58,59}$. In brief, Zn-Mn and Zn-Co ferrite nanoparticles are prepared by hydrothermal coprecipitation of aqueous solutions of $\mathrm{Fe}^{3+}, \mathrm{Zn}^{2+}$ and $\mathrm{Mn}^{2+}$ or $\mathrm{Co}^{2+}(0.5 \mathrm{~mol} / \mathrm{L})$ in strong alkaline medium $(\mathrm{NaOH} 2$ $\mathrm{mol} / \mathrm{L})$ at $100{ }^{\circ} \mathrm{C}$ under vigorous stirring. Aiming the obtention of stoichiometric mixed ferrite core $\left(\mathrm{Zn}_{\mathrm{x}} \mathrm{M}_{(1-\mathrm{x})} \mathrm{Fe}_{2} \mathrm{O}_{4}\right.$, $\mathrm{M}$ being $\mathrm{Mn}$ or $\mathrm{Co}$ ), the metallic salts mixtures $\mathrm{Zn}: \mathrm{M}: \mathrm{Fe}$ are tuned as $\mathrm{x}:(1-\mathrm{x}): 2$ ( $\mathrm{x}$ varying between $0.1-0.9)$. Next, the as prepared precipitate is cleaned and acidified with $\mathrm{HNO}_{3}$ $2 \mathrm{~mol} / \mathrm{L}$ to reverse the surface charge of the NPs and eliminate undesirable by-product. At this stage, the release of the metallic cations from the surface is more significant for the divalent metals when compared to $\mathrm{Fe}^{3+}$, indicating the beginning of the iron-rich shell formation onto the NPs surface $^{59}$. As the chemical stability of the NPs in acidic medium is achieved by incorporating more iron atoms onto the surface, the precipitate is hydrothermally treated with a $0.5 \mathrm{~mol} / \mathrm{L} \mathrm{Fe}\left(\mathrm{NO}_{3}\right)_{3}$ solution at $100{ }^{\circ} \mathrm{C}$ for $15 \mathrm{~min}$. Finally, the particles are peptized by $\mathrm{pH}$ and ionic strength adjustment in the aqueous medium, to control the surface charge density and the screening of the electrostatic repulsion between particles to ensure colloidal stability. The NPs of varying composition are indexed as ZC1 to ZC9 for core/shell NPs based on Zn-Co ferrite cores and ZM1 to ZM9 for those obtained with Zn-Mn ferrite ones.

\section{B. Chemical, Structural and Morphological Charac- terization}

Chemical composition of NPs is checked by determination of $\mathrm{Zn}, \mathrm{Mn}$, Co and Fe concentrations with EnergyDispersive X ray spectroscopy (EDX) and Atomic Absorption Spectroscopy technique (AAS). Both measurements are performed at Universidade de Brasília (UnB) with a Shimadzu EDX 720HS and with a Thermo Scientific $®$ Spectrometer model S Series AA, respectively. For AAS measurements, specific lines are chosen for each metal: Fe $(248.3 \mathrm{~nm} / 372.0 \mathrm{~nm}), \mathrm{Zn}(213.9 \mathrm{~nm}), \mathrm{Mn}(279.5 \mathrm{~nm})$ and Co $(240.7 \mathrm{~nm})$ to avoid interference effects.

The crystalline structure of NPs is investigated by X Ray Powder Diffraction (XRPD) after evaporation of the liquid phase from the samples. For samples based on $\mathrm{Zn}-\mathrm{Mn}$ ferrites, measurements are carried out at Laboratório Nacional de Luz Síncrotron (LNLS) using the D12A-XRD1 beamline and a x ray wavelength $\lambda=1.77155 \AA$ in the $2 \theta$ range $15^{\circ}-120^{\circ}$. For Zn-Co ferrites based samples, diffractograms are collected using a D8 Focus (Bruker) Diffractometer at $\mathrm{UnB}$, using the $\mathrm{Cu}-\mathrm{K} \alpha$ radiation with $\lambda=1.5406$ $\AA$ in the $2 \theta$ range $20^{\circ}-80^{\circ}$. The diffracted lines are related to the characteristic interplanar spacings of the structure 
and compared with bulk standard data of International Centre for Diffraction Data (ICDD) for $\mathrm{MnFe}_{2} \mathrm{O}_{4}$ (ICDD no. 01-073-1964), $\mathrm{ZnFe}_{2} \mathrm{O}_{4}$ (ICDD no. 98-007-2033), $\mathrm{CoFe}_{2} \mathrm{O}_{4}$ (ICDD no. 00-022-1086), $\mathrm{Zn}_{\mathrm{x}} \mathrm{Mn}_{(1-\mathrm{x})} \mathrm{Fe}_{2} \mathrm{O}_{4}$ (ICDD no. 98002-8512 to 8516), $\mathrm{Zn}_{\mathrm{x}} \mathrm{Co}_{(1-\mathrm{x})} \mathrm{Fe}_{2} \mathrm{O}_{4}$ (ICDD no. 98-016-6201 to 6204), $\gamma-\mathrm{Fe}_{2} \mathrm{O}_{3}$ (ICDD no. 98-008-7119) and $\mathrm{Fe}_{3} \mathrm{O}_{4}$ (ICDD no. 98-002-6410). Crystalline sizes are obtained using Scherrer's formula $D_{X R}=0.9 \lambda / \beta \cos \theta$ applied to the most intense peak [311], $\beta$ being its width at half maximum (intrinsic width is discounted using a Si standard).

For all samples, morphology, crystallinity and size distributions of NPs are investigated by Transmission Electron Microscopy (TEM - JEOL JEM100 CX II) and High Resolution TEM (HRTEM - JEOL JEM2010), both with a LaB6 filament operated at $200 \mathrm{kV}$, carried out at Université Pierre et Marie Curie (UPMC). Size distribution histograms are obtained by counting approximately 600 particles and are fitted by a lognormal distribution $P(D)=\exp \left(-\ln ^{2}\left(D / D_{0}\right) / 2 s^{2}\right) / \sqrt{2 \pi} D s, D_{0}$ and $s$ being the characteristic diameter and the polydispersity index, respectively. Moreover, the interplanar spacings associated to the observed reticular planes of the spinel cubic structure can be determined by Fast Fourier Transform (FFT) analysis and compared to ICDD data. In order to go further in detail on the NPs' core/shell structure, sample ZM5 is probed by HRTEM using a TECNAI F30 (FEI Company) microscope equipped with a Field Emission Gun (FEG) operated at $300 \mathrm{kV}$ in Laboratorio de Microscopías Avanzadas (LMA) - University of Zaragoza (UZ). Z-contrast imaging is obtained using Scanning Transmission Electron Microscopy (STEM) mode with a High-Angle Annular Dark Field (HAADF) and with an Oxford ultra-thin window EDX detector to test the chemical composition profile.

\section{Magnetic Measurements}

Magnetization measurements are performed at UnB (Instituto de Física) and at UPMC (MONARIS Laboratory) using the same model of Vibrating Sample Magnetometer (VSM - PPMS, Quantum Design mod. 6000). Magnetization curves are obtained as a function of the applied field (up to $7160 \mathrm{kA} / \mathrm{m}$ ), at $5 \mathrm{~K}$ after a Zero Field Cooling (ZFC) procedure and at $300 \mathrm{~K}$ for dilute magnetic fluids (MF) samples $(\sim 10 \mathrm{mg} / \mathrm{mL})$. In this regime of negligible magnetic dipolar interactions and at room temperature, the reversible curves are analyzed using Langevin contributions of spheroidal particles weighted by a log-normal distribution of NPs sizes ${ }^{58}$. Magnetic colloids can be considered as a gas of non-interacting particles ${ }^{62,63}$ which presents characteristic giant paramagnetic behavior, so that the magnetization can be expressed by:

$$
\frac{\sigma}{\sigma_{S}}=\frac{M}{M_{S}}=\frac{\int_{0}^{\infty} d^{3} L_{1}\left[\xi\left(D, s_{D}\right)\right] P(D) d D}{\int_{0}^{\infty} d^{3} P(D) d D},
$$

where $\sigma$ is the magnetization per unit mass $\sigma=M / \rho_{N P}$, $M$ being the magnetization per unit of volume, $\rho_{N P}$ the NP's density (see E.S.I), $\sigma_{s}$ the saturation magnetization per unit mass $\sigma_{s}=M_{S} / \rho_{N P}, M_{S}$ the saturation magnetization of the NP of moment $\mu=M_{s} V, \xi=\mu_{0} \mu H / k_{B} T$ the Langevin parameter with $\mu_{0}$ being the permeability constant and
$L_{1}=\operatorname{coth} \xi-1 / \xi$ the first Langevin function. In the high field limit $\mu_{0} \mu H / k_{B} T \gg 1$ the magnetization follows a linear dependence on $H^{-1}$, thus allowing the determination of $\sigma_{s}$, by performing a linear extrapolation of $\mathrm{H}^{-1}$ in the high magnetic field region.

From hysteresis loops of ZFC MFs, obtained at $5 \mathrm{~K}$, we determine the high field magnetization at this temperature and the coercive field $H_{C}(5 \mathrm{~K})$. The effective magnetic anisotropy constants $K_{e f}=\mu_{0} M_{s} H_{a} / 2$ are associated to $E_{a}=K_{e f} V, H_{a}$ being the anisotropy field, is here deduced assimilating $H_{C}(5 \mathrm{~K})$ to $H_{a} / 2$, its maximum value (to be reached at $E_{a} / k_{B} T \gg 1$ with this ensemble of randomly oriented NPs). It is then possible to compare together the respective magnetic hardnesses of the various NPs.

Finally, for both Zn-Mn and Zn-Co based ferrites samples, we measure the thermal dependence of the magnetization of pressed powder samples ( $\sim 2$ Ton) obtained at 80 $\mathrm{kA} / \mathrm{m}$, above room temperature up to $600 / 700 \mathrm{~K}$ using an oven coupled to the VSM at UnB. Curie temperature is obtained by the extrapolation in temperature of the magnetization $\quad \sigma \rightarrow 0$. The thermomagnetic coefficient $k_{T M}=-\Delta \sigma / \Delta T$ is determined at $80 \mathrm{kA} / \mathrm{m}$ in the temperature range of interest for hyperthermia applications $(300$ to $350 \mathrm{~K}$ ).

\section{Specific Power Absorption Measurements}

Power absorption experiments are taken at INA - UZ using a commercial device (DM100 from nB nanoScale Biomagnetics) device to determine the Specific Power Absorption (SPA) of ferrofluid samples. In adiabatic systems, the amount of heat released by the NPs (power losses) is equal to the power absorbed and is given by the area enclosed by the AC dynamic hysteresis loop ${ }^{5}$. Thus, the SPA (W $\mathrm{g}^{-1}$ ) index is defined as $S P A=P / c_{N P}$, with $P\left(\mathrm{~W} \mathrm{~m}^{-3}\right)$ being the volumetric power loss and $c_{N P}$ the concentration of magnetic NPs within the solution in $\mathrm{mg} / \mathrm{mL}$. It is experimentally determined by:

$$
S P A=c_{\text {solvent }} \frac{m_{\text {solvent }}}{m_{N P}} \frac{\Delta T}{\Delta t},
$$

where $c_{\text {solvent }}$ is the specific heat of the solvent, $m_{\text {solvent }}$ and $m_{N P}$ are respectively the mass of the solvent and the NPs and $\Delta T / \Delta t$ is the initial slope of experimental heating curve. For all the samples, measurements are carried out at NPs' concentration around $10 \mathrm{mg} / \mathrm{mL}$ (aiming to minimize changes in collective behavior due to dipolar interactions between NPs) using an AC magnetic field amplitude of 12 $\mathrm{kA} / \mathrm{m}$ at a frequency of $265 \mathrm{kHz}$. Data are also collected for frequencies between 265 and $831 \mathrm{kHz}$ at $12 \mathrm{kA} / \mathrm{m}$ for $\mathrm{MF}$ based on ZC1 NPs, and varying the field amplitudes in the range of $4-24 \mathrm{kA} / \mathrm{m}$ for the $\mathrm{MF}$ samples based on $\mathrm{ZM} 2$, ZM3, ZM6 and ZM9 NPs.

\section{RESULTS}

\section{A. Core/Shell Nanoparticles}

We synthesized mixed ferrites NPs with a core/shell structure portrayed as $\mathrm{Zn}_{\mathrm{x}} \mathrm{M}_{\mathrm{y}} \mathrm{Fe}_{\mathrm{z}} \mathrm{O}_{4} @ \gamma-\mathrm{Fe}_{2} \mathrm{O}_{3}$ ( $\mathrm{M}$ being $\mathrm{Mn}$ or $\mathrm{Co})$, to disperse them in acidic aqueous medium $(\mathrm{pH} \sim$ 
2). The NPs surface modifications induced by the surface treatment and the formation of the core/shell structure are well followed by EDX and AAS measurements of metal concentrations at each step of the synthesis [ref 58 e 59]. Table S1 collect the chemical characteristics of both bare NPs and surface-treated ones. The results show that, after the coprecipitation step, the stoichiometry of bare NPs does not correspond to that of an ideal ferrite. For each type of sample, the fractions of $(\mathrm{Zn}+\mathrm{Mn})$ and $(\mathrm{Zn}+\mathrm{Co})$ decreases with increasing $\mathrm{Zn}$ substitution, from about 0.33 , the exact value of an ideal ferrite, obtained at low $\mathrm{Zn}$ content, to much smaller ones. Thus we write the chemical formula as $\mathrm{Zn}_{\mathrm{x}} \mathrm{M}_{\mathrm{y}} \mathrm{Fe}_{(3-\mathrm{x}-\mathrm{y})} \mathrm{O}_{4+\delta}$ to obtain the stoichiometry of bare NPs. An excess of positive charges coming from two mechanisms exactly balances the extra electronegative charge appearing per formula unit. The first one occurs in both kinds of samples and is related to the lower zinc content and to the larger iron content when compared to the expected nominal values. Thus, the substitution of a divalent ion by a trivalent one leads to an excess of positive charges. Moreover, in Mn based Nps, mixed valence states of $\mathrm{Mn}$ ions have also to be considered, as oxidation of $\mathrm{Mn}$ ions would occur during the synthesis in alkaline medium. Very recently, we studied in details the local structure of core/shell NPs based on pure Mn-ferrite core elaborated by the same synthesis procedure ${ }^{64}$. The oxidation state of Mn ions has been investigated by using X Ray Absorption Spectroscopy. It is found to be 2.9 in bare NPs, a value that we will use here. More details on the stoichiometry calculations are given in the SI. Table S1 lists the values of the oxidation degree $\delta$ and the chemical formula obtained as described and renormalized in order to correspond to a close-packed arrangement of 32 oxygen anions. It therefore enhances the existence of metal ions vacancies randomly distributed in both tetrahedral and octahedral interstitial sites. A detailed analysis of structural studies of our mixed ferrite based NPs is on progress and will be published elsewhere.

After the surface treatment, the fractions of $(\mathrm{Zn}+\mathrm{Mn})$ and $(\mathrm{Zn}+\mathrm{Co})$ also decreases. This is a direct consequence of both the removal of divalent metal cations from the NPs surface during the acid cleaning and the incorporation of more iron ions into the surface layer during the surface treatment with ferric nitrate ${ }^{59}$. This effect is more pronounced for Zn-Mn ferrite NPs, mainly due to their higher surface/volume ratio as the NP size becomes smaller for larger Zn content. The fractions of maghemite shell deduced as shown in the SI from the metal concentrations determined after the surface treatment are collected in Table S1.

XRPD patterns of representative powder samples, all exhibiting characteristic peaks of cubic spinel structure, are seen in Figure S1-a and Figure S1-b. Samples' characteristics such as size, lattice parameter and density obtained from these diffractograms, as well as information about the procedure are also found in the SI. The cubic cell size varies with $\mathrm{Zn}$ content for both sets of samples. They are compared to the lattice parameters calculated using Vegard's law. The existence of a maghemite surface layer induces an overall decrease of the lattice parameters, whose tendencies still follow the theoretically predicted ones (see Figure S2). Both Zn-Co and Zn-Mn ferrites nanoparticles present a decrease of the crystalline size as $\mathrm{Zn}$ fraction increases and this effect is more pronounced for $\mathrm{Zn}-\mathrm{Mn}$ ferrites (see Table S2), in good agreement with previous reports $49,50,53,65,66$. This indicates that the nucleation process would be favored in preference to the crystalline growth during the coprecipitation ${ }^{67,68}$. Moreover, local structure must be considered, more particularly in $\mathrm{Zn}-\mathrm{Mn}$ based samples. As an example, interatomic distances Mn-O are larger than $\mathrm{Zn}-\mathrm{O}$ counterparts and should lead to a reduction of the cubic cell ${ }^{69}$ therefore inducing smaller NPs sizes. The oxidation of $\mathrm{Mn}$ ions and the location of the metallic cations at interstitial sites may also contribute to a decrease of both cell size and NPs diameter ${ }^{64,70}$.
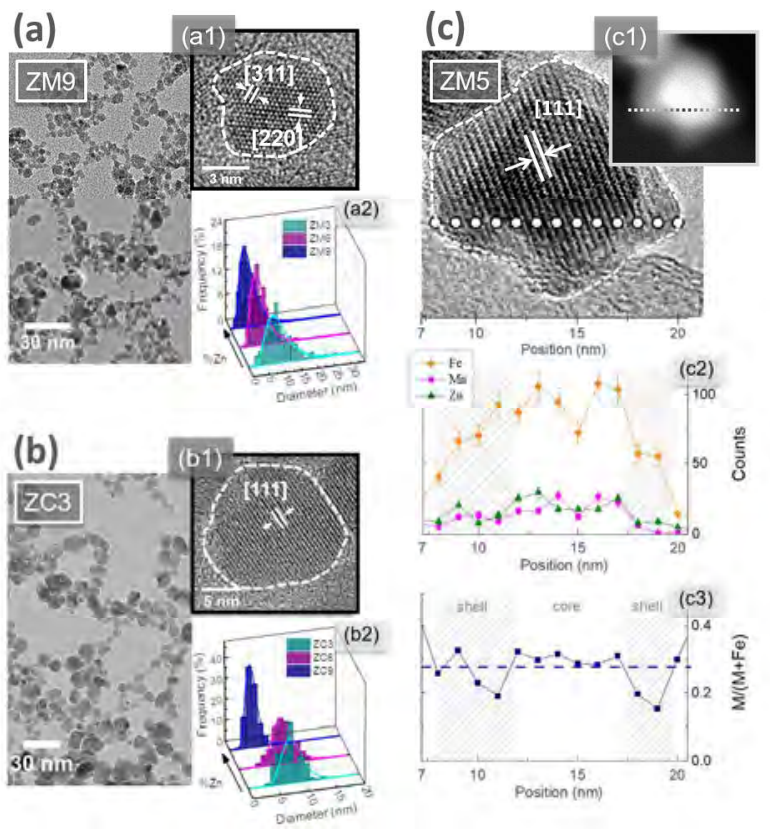

Figure 1. (a) and (b) TEM micrograph for ZM9 and ZC3 samples. (a1) and (b1) are the high-resolution micrographs with some crystalline planes of spinel structure identified; (a2) shows the histograms and corresponding fit of log-normal distribution for ZM3, ZM6 and ZM9 NPs; (b2) same for ZC3, ZC6 and ZC9 NPs; (c) HRTEM of representative nanoparticle; (c1) STEM of same NP presented in (c); (c2) Mn, Zn and Fe STEM profile of a single core/shell NP for the same NP; and (c3) is the core/shell model applied to the graph (c2).

Conventional TEM images (see Figure 1a and Figure 1b) show well crystalline "rock-like" shape NPs (See HRTEM in Figure 1a1 and Figure1b1). The corresponding size histograms are shown in Figure $1 \mathrm{a} 2$ and Figure 1b2. Results are listed in Table S2. As already observed for crystalline XRPD sizes, both Zn-Co ferrites and Zn-Mn ferrites present a downward change of characteristic diameter with increasing Zn substitution, more intense for $\mathrm{Zn}$-Mn ferrites.

Moreover, the core/shell nature of our NPs is clearly verified in sample ZM5 by using FEG-HRTEM measure- 
ments (Figure 1c), whose larger contrast allowed us to observe regions of the same nanocrystal with different intensities, pointing towards the existence of a thin and uneven shell. We further investigate this issue using a STEM-HAADF image of the same NP (Figure 1c1). The contrast between the regions of the sample provides information about the morpho-chemical composition. Then, the chemical composition profile is obtained for this representative NP and it is depicted in Figure 1c2 and Figure $1 \mathrm{c} 3$. We delimit the surface layer in the presented graphs in order to clarify the analysis. Figure $1 \mathrm{c} 2$ show high concentrations of $\mathrm{Fe}$ and almost zero for $\mathrm{Zn}$ and $\mathrm{Mn}$ in the shaded area. It also indicates that the transition at the interface with the core is rather smooth. In Figure 1c3, we present the ratio between the sum of the intensities relative $\mathrm{Zn}$ and $\mathrm{Mn}$ and that relative to the total metals. It is expected to be 0.33 for a stoichiometric Zn-Mn ferrite. In the demarked core region, this ratio is constant around 0.3 , in agreement with the value 0.28 , predicted by the chemical analysis presented in Table S1. In both shell regions, we observe a decrease of this value, clearly indicating the ironenriched surface. We have also verified the core/shell chemical structure for ZC3 sample, as depicted in Figure S3. Even though the surface layer is not homogeneous and presents some remaining content of core divalent metals in the composition, the obtention of the core-shell structure by hydrothermal soft chemistry is remarkable. Indeed, our chemical core/shell approach well accounts for several structural and magnetic properties, such as coordination of core and shell metal ions ${ }^{71}$ and intrinsic NPs' magnetization ${ }^{63,72,73}$.

\section{B. Magnetic Characterization of NPs at $300 \mathrm{~K}$ and $5 \mathrm{~K}$}

Figure 2a and Figure 2b show typical room temperature magnetization curves obtained for dilute magnetic fluids based on ZM9 and ZC8 NPs. At room temperature, because of the rotational degrees of freedom of their NPs in the fluid carrier, the magnetic fluid samples present a giant paramagnetism behavior. Their $\mathrm{M}(\mathrm{H})$ curves thus show no coercive field. In main Figure 2a (resp. Figure 2b), the solid line represents the best magnetization fit calculated using Eq. 1. From the linear fit at high-field (insets of Figure 2a and Figure 2b) we deduce the saturation magnetization values $\sigma_{s}(300 \mathrm{~K})$ given in Table S3. They are presented as a function of the zinc content in Figure $3 \mathrm{a}$ and Figure $3 \mathrm{~b}$.
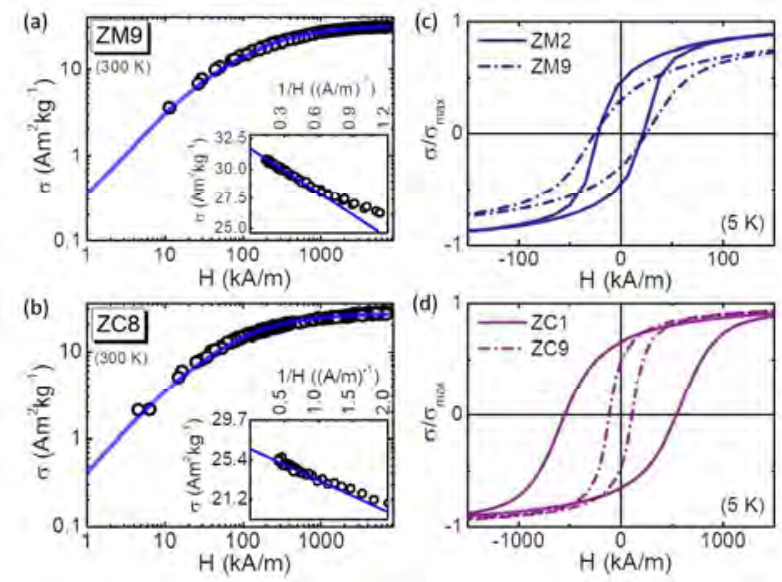

Figure 2. (a) and (b) magnetization data as a function of the applied field at $300 \mathrm{~K}$ (open circles) and best Langevin fit (solid lines) for ZM9 and ZC8 samples in dilute regime. Insets show the high field analysis to determine $\sigma_{s}$. Hysteresis cycles for $\mathrm{Zn}-\mathrm{Mn}(\mathrm{c})$ and $\mathrm{Zn}-\mathrm{Co}(\mathrm{d})$ samples recorded at $\mathrm{T}=5 \mathrm{~K}$.
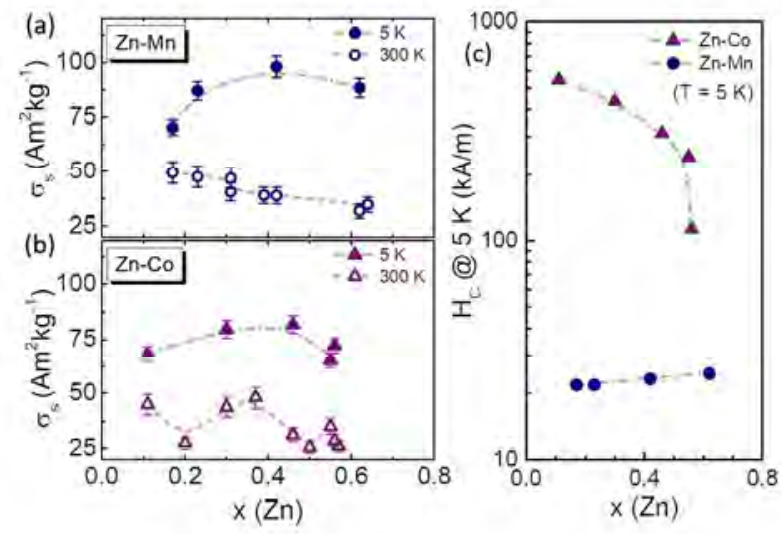

Figure 3. (a) and (b) Saturation magnetization of NPs in dilute regime at $300 \mathrm{~K}$ (open symbols) and $5 \mathrm{~K}$ (filled symbols) for $\mathrm{Zn}-\mathrm{Mn}$ and $\mathrm{Zn}$-Co samples, respectively (c) Coercive field $H_{C}$ obtained at $5 \mathrm{~K}$ as a function of $\mathrm{Zn}$ substitution, for $\mathrm{Zn}$-Co and $\mathrm{Zn}-\mathrm{Mn}$ series, both in dilute regime (error bars in $H_{C}$ are not represented since they are about $1.6 \mathrm{kA} / \mathrm{m}$ ).

At room temperature, these same NPs, mechanically blocked in disorder in pressed powder samples, all present a small coercive field $\left(H_{c}<9 \mathrm{kA} / \mathrm{m}\right)$ - see Figure S5.

At very low temperature, in Zero-Field-Cooled (ZFC) MF, the magnetic NPs are also mechanically blocked and the magnetization process leads to a hysteresis loop well described by a Stoner-Wohlfarth formalism for ferromagnetic $\mathrm{NPs}^{74}$. Figure $2 \mathrm{c}$ and Figure $2 \mathrm{~d}$ show typical magnetization hysteresis loops recorded at $5 \mathrm{~K}$ with ZFC-MF samples based on ZM2, ZM9, ZC1 and ZC9 NPs. The characteristic saturation magnetization $\sigma_{s}(5 \mathrm{~K})$ and the coercive field $H_{c}$ $(5 \mathrm{~K})$ are plotted as a function of the zinc content $\mathrm{x}$ in Figure 3. Both series of samples based on $\mathrm{Zn}-\mathrm{Mn}$ and $\mathrm{Zn}-\mathrm{Co}$ ferrites, have the same qualitative behavior of $\sigma_{s}(5 \mathrm{~K})$ with 
the zinc content. It well compares with the well-known variations obtained for $\mathrm{Zn}$ mixed ferrite bulk materials ${ }^{75}$. Although the NPs cores are here surrounded by a surface shell of maghemite, the magnetization still presents a typical maximum with $\mathrm{Zn}$ content around $\mathrm{x}=0.5$.

The magnetization decrease observed for larger $\mathrm{x}$ values can be understood as in bulk materials. The preference of the diamagnetic $\mathrm{Zn}$ ions for tetrahedral sites is responsible for the weakening of the A-B super-exchange interaction with increasing $\mathrm{Zn}$ concentration ${ }^{75,76}$. In the present core/shell NPs, this decrease is smoother and several reasons could account for this behavior. First, the chemical composition of the NPs is not homogeneous, with a maghemite surface shell surrounding a non-stoichiometric core, this effect being more pronounced for large zinc content. Second, there is a supplementary contribution to the NP magnetization due to the disordered surface-spins freezing at very low temperatures ${ }^{72,73}$, mostly observed for smaller NPs (those with the larger zinc content). At $300 \mathrm{~K}$, the magnetization is smaller, as expected, and for $\mathrm{Zn}-\mathrm{Mn}$ samples, slightly decreases with zinc percentage. The difference between $\sigma_{s}$ values at $5 \mathrm{~K}$ and $300 \mathrm{~K}$ increases with $\mathrm{Zn}$ content. This could be due to finite-size effects which would modify the thermal dependence of the NPs magnetization $^{72}$.

From hysteresis loops of Figure $2 \mathrm{c}$ and Figure $2 \mathrm{~d}$ we deduce the coercive field $H_{C}$ at $5 \mathrm{~K}$ and their values are presented against $\mathrm{Zn}$ substitution in Figure 3c for both $\mathrm{Zn}-\mathrm{Mn}$ and Zn-Co series of samples. For Zn-Co series the $K_{e f}$ values (see Table S3) well compare with that of cobalt ferrite $^{77}$ around $3.53 \times 10^{5} \mathrm{~J} / \mathrm{m}^{3}$. They also decrease by almost one order of magnitude from $2.5 \times 10^{5}$ to $5.5 \times 10^{4}$ $\mathrm{J} / \mathrm{m}^{3}$ with increasing $\mathrm{Zn}$ content. It reflects the incorporation of less anisotropic ions in the structure of NPs' core. On the other hand, for Zn-Mn series the deduced $K_{e f}$ values can be also compared to those of Mn ferrite bulk materials $^{78}\left(2.50 \times 10^{3} \mathrm{~J} / \mathrm{m}^{3}\right)$. They are here larger and they augment with the $\mathrm{Zn}$ content, from 1.0 to $1.5 \times 10^{4} \mathrm{~J} / \mathrm{m}^{3}$. This is most probably due to a surface contribution (as in maghemite ${ }^{79}$ ) associated to the NPs size ${ }^{80}$, which is smaller with increasing zinc concentration. As expected, the NP anisotropy constants of Zn-Co ferrite based NPs, are much larger than those of Zn-Mn ferrite based NPs. As magnetic anisotropy affects Néel relaxation and as we deal here with a large range of anisotropy values, we expect to probe the relation between both magnetic hardness and exchange coupling between hard/soft and soft/soft core/shell NPs on the heat generation performance.

\section{Curie Temperature and Thermomagnetic Coefficient}

The thermal dependence of the magnetization $\sigma$ at 80 $\mathrm{kA} / \mathrm{m}$ of NPs' pressed powders is illustrated with ZM9 and ZC9 NPs in Figure S4. For both kinds of samples, the magnetization decreases with increasing temperatures as expected. This thermal dependence allows to determine the Curie temperature $T_{C}$ and the thermomagnetic coefficient $k_{T M}$. Both characteristics are obtained by linear extrapolation of $\sigma$ as a function of temperature. Their

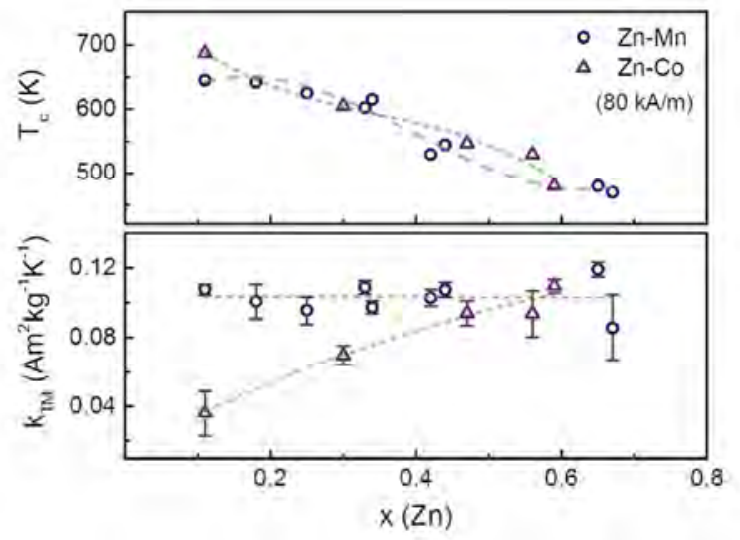

Figure 4. NPs Curie temperature as a function of Zn substitution for the entire series of $\mathrm{Zn}-\mathrm{Co}$ and $\mathrm{Zn}-\mathrm{Mn}$ ferrite powder samples and thermomagnetic coefficients averaged in the range of temperature $300-350 \mathrm{~K}$ (error bars are standard deviation from the average).

respective values are plotted as a function of the $\mathrm{Zn}$ content in Figure 4. In Zn-Mn samples, $T_{C}$ decreases from 645 $\mathrm{K}$ to $480 \mathrm{~K}$ when the amount of $\mathrm{Zn}$ in the NPs' core increases from $x=0.11$ to $x=0.65$. In the investigated range of $\mathrm{Zn}$ composition, the values found here are larger than those extracted from the literature for NPs of stoichiometric Mn$\mathrm{Zn}$ ferrite ${ }^{49,50,68,81}$. It should be related first to the chemical composition of the core, which is non-stoichiometric with Zn loss and Fe enrichment. Second, the presence of the surface shell of maghemite should also lead to an increase of the $T_{C}$ of the core/shell NPs since for maghemite bulk materials $T_{C}$ lies in the range 820 - $986 \mathrm{~K}^{82}$, a value much larger than that of $\mathrm{Mn}$-Zn ferrites. In Zn-Co ferrite based samples $T_{C}$ decreases from $687 \mathrm{~K}$ to $480 \mathrm{~K}$ for increasing Zn substitution from $x=0.11$ to $x=0.59$. Such values are also larger than those commonly observed in pure Zn-Co ferrite NPs ${ }^{49,83}$ and reflects once more the combined effects of non-stoichiometry of the core composition and surrounding maghemite shell.

Figure 4 also depicts, as a function of Zn substitution, the values of the thermomagnetic coefficient $k_{T M}(=-\Delta \sigma / \Delta \mathrm{T})$ obtained in the presence of an external field of $80 \mathrm{kA} / \mathrm{m}$ and averaged over the T-range $300-350 \mathrm{~K}$. For the Zn-Mn series, $k_{T M}$ is approximately constant independent of the Zn content (except for ZM9 powder sample for which it slightly decreases). These measured values are smaller than those of pure Zn-Mn ferrite NPs ${ }^{49,50}$. For Zn-Co ferrite NPs, $k_{T M}$ increases with Zn content, nonetheless these values are approximately three times lower than those reported in the literature ${ }^{49,83}$. The smaller values observed in the two series of samples are closely related to iron enrichment in both core and shell phases. Indeed, the Curie temperature of maghemite is larger than that of $\mathrm{Zn}$ substituted ferrite materials leading to a smoother decrease of the magnetization with temperature. Moreover, since the applied field is too low to saturate the core of the NPs, the different behavior between both series might be related to the magnetic anisotropy, thus increasing the thermomagnetic coefficient with $\mathrm{Zn}$ content for $\mathrm{Zn}-\mathrm{Co}$ 
samples. As seen in Figure S5, powder samples ZC1 to ZC4 and ZM9 show the largest coercive fields at $300 \mathrm{~K}$ and also the smallest values of thermomagnetic coefficient. For softer magnetic NPs, the thermal energy would disorient the magnetic moments more efficiently, decreasing the magnetization more abruptly.

\section{Specific Power Absorption}

The SPA results for some representative NPs dispersed in aqueous medium are presented in Figure 5 as a function of both magnetic field amplitudes and frequencies. They are analyzed in the framework of the Linear Response Theory (LRT), which calculates the dynamic hysteresis area by assuming that the magnetic response of an assembly of magnetic NPs is linear with the external field 5 . The validity of LRT takes place when $\xi \ll 1$, which is satisfied when the amplitude of the applied magnetic field is much smaller than the anisotropy field of the NPs $\left(H_{0} \ll H_{a}\right)^{6}$ (it is true here as $E_{a} / k_{B} T \geq 1$ for all the magnetic fluid samples at $300 \mathrm{~K}$ ). Then, in such conditions and for the randomly orientated magnetic NPs, the density of absorbed power is given by 5 :

$$
P=\frac{1}{2} \mu_{0} \chi_{0} H_{0}^{2} \frac{(2 \pi f)^{2} \tau}{1+(2 \pi f \tau)^{2}},
$$
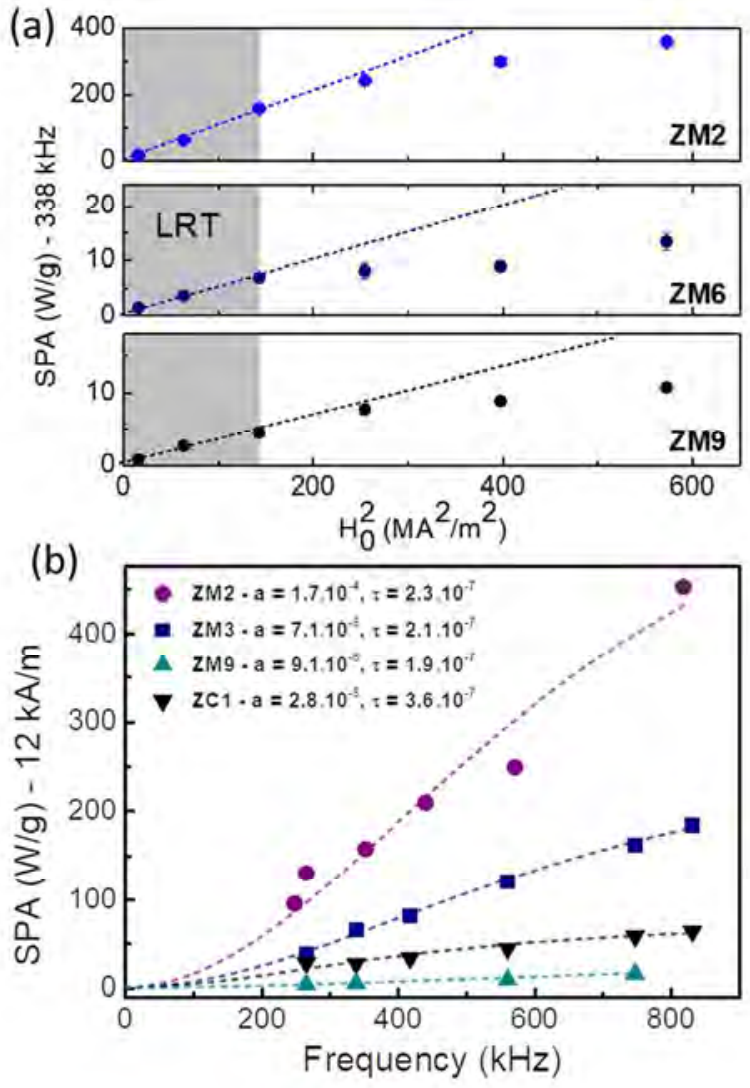

Figure 5. (a) Range of linearity of SPA (338 kHz) with $H_{0}^{2}$ for ZM2, ZM6 and ZM9 NPs, both dispersed in aqueous medium. Grey zones indicate the range of validity of LRT; (b) Frequency dependence of SPA $(12 \mathrm{kA} / \mathrm{m})$. The fit represents the frequency dependence of SPA in the framework of LRT. $\chi_{0}=\mu_{0} M_{s}^{2} V / 3 k_{B} T$ being the initial static susceptibility. Figure 5a shows the SPA values as a function of the square of the magnetic field amplitude for ZM2, ZM6 and ZM9 samples. One can distinguish two regions, the first one up to $H_{0} \sim 12 \mathrm{kA} / \mathrm{m}$, where the SPA is proportional to $H_{0}^{2}$, and where the Eq. 3 may apply. For larger $H_{0}$ values, SPA determinations deviate from LRT.

Let us precise the domain of validity of the LRT for the different NPs with different hardnesses. If the condition $H_{0} \ll H_{a}$ is valid for $H_{0}=12 \mathrm{kA} / \mathrm{m}$ with ZM2 NPs, which are the less anisotropic among the two series of NPs, it will also stand for the other ones, which are all magnetically harder. Figure $5 \mathrm{~b}$ depicts the frequency dependence of SPA at $H_{0}=12 \mathrm{kA} / \mathrm{m}$ for ZC1, ZM2, ZM3 and ZM9 NPs. Data are fitted using Eq. 3 with two free parameters: $\mathrm{a}=\frac{1}{2} \mu_{0} \chi_{0} H_{0}^{2}$ and $\tau$. The best fit (dashed lines) values shown in the figure well compare to those reported in the literature ${ }^{11,12}$.

It is worth noting that, considering only Néel relaxation, the $\tau$ values could be translated into magnetic anisotropy constants at $300 \mathrm{~K}$. They are in agreement with the $\mathrm{K}$ values calculated at $300 \mathrm{~K}$ from the data obtained in the low temperature experiments using the methodology applied by Garaio et $\mathrm{al}^{84}$. Future studies intend to investigate more precisely, in these core/shell NPs, the combined effect of size variations and anisotropy on the relaxation times

To compare all the samples investigated here assuring LRT to be valid in all samples, the measurements have been performed at $f=265 \mathrm{kHz}$ and $H_{0}=12 \mathrm{kA} / \mathrm{m}$, since in this regime all the samples follow the LRT. The results are collected as a function of $\mathrm{Zn}$ content for both series in Figure 6.

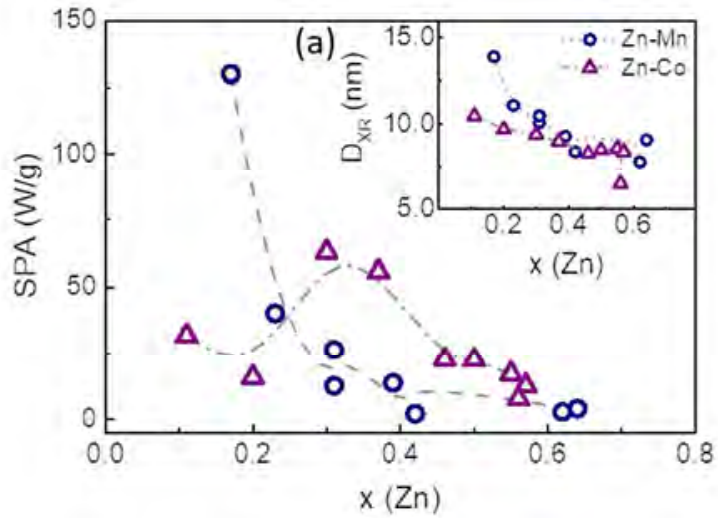

Figure 6. SPA values obtained at $12 \mathrm{kA} / \mathrm{m}$ and $265 \mathrm{kHz}$ for the entire $\mathrm{Zn}-\mathrm{Mn}$ and $\mathrm{Zn}$-Co series as a function of $\mathrm{Zn}$ content $(\mathrm{x})$ (a) the inset depicts the variation of the NPs crystalline diameter with $\mathrm{x}$.

In the case of NPs based on Zn-Mn core, those with the lower $\mathrm{Zn}$-content exhibit the best efficiency for heat generation $(\mathrm{SPA}=130 \mathrm{~W} / \mathrm{g}$ for $\mathrm{x}=0.18)$ and the SPA almost vanishes for larger values of $\mathrm{Zn}$ substitution. In this series with negligible variations of magnetic anisotropy, this feature seems to be most likely related to the decrease of 
both NPs' diameter and saturation magnetization at $300 \mathrm{~K}$ with Zn substitution (see Figure 6a and inset of Figure 3a). For Zn-Co series, SPA values are at the same level $(\sim 20$ $\mathrm{W} / \mathrm{g}$ ) than those obtained for $\mathrm{Zn}-\mathrm{Mn}$ series with $\mathrm{x} \geq 0.25$, except for ZC3 and ZC4 samples, which reach a maximum around $60 \mathrm{~W} / \mathrm{g}$. The presence of this maximum is most likely related to the combination of several characteristics, which will be discussed in detail in the following.

\section{DISCUSSION}

We discuss here the performances of the studied core/shell NPs based on Zn-Mn and Zn-Co ferrite cores, focusing mainly on heat generation phenomena while dispersed in an aqueous medium. As described above, these NPs present SPA values comparable to those previously reported with similar ferrite NPs dispersed in water ${ }^{11,12,23,85}$. The increase of $\mathrm{Zn}$ content induces the weakening of the superexchange interactions and affects not only the saturation magnetization but also the Curie temperature and power absorption. These NPs could therefore be good candidates for magnetohyperthermia treatments in a more safety strategy since it would help to avoid overheating and the necrosis of healthy tissues. It is worthwhile to remark that NPs' efficiency on heat generation is affected by the diameter of the NPs, magnetic anisotropy constant and saturation magnetization, all parameters that were carefully determined in previous sections.

Figure 7a depicts SPA as a function of the NPs' crystalline diameter for samples with similar Mn or Co content (y index ranging between 0.67 to 0.48 ) and $\sigma_{s}$, in each $\mathrm{Zn}-\mathrm{Mn}$ and $\mathrm{Zn}$-Co series. We observe that the SPA values, which are approximately the same for both kind of samples, are obtained in different ranges of diameters. It illustrates the role of the magnetic anisotropy constant on the heat generation efficiency, which shifts the value of the NP diameter that corresponds to the maximum of the SPA ${ }^{5,6}$ defined by $2 \pi f \tau=1$. However, an increment of anisotropy constant leads to an increase of $\tau_{N}$, and consequently $\tau$, but not necessarily maximizes the heating efficiency. Indeed, for highly anisotropic NPs, the magnetic moment is strongly coupled with the anisotropy field and preferentially relaxes by mechanical rotation. In this case, larger SPA will be obtained for smaller NPs when compared to lower anisotropic ones. In such scenario, an increment of media viscosity, such as often occurs in biological media, will hamper the Brownian motion, decreasing the heat generation efficiency of the $\mathrm{NPs}^{23}$. Moreover, the larger SPA values observed for ZC3 and ZC4 samples have probably to be attributed to the large values of saturation magnetization (see Table S3) conjugated with the lowering of the anisotropy barrier induced by increasing zinc content.
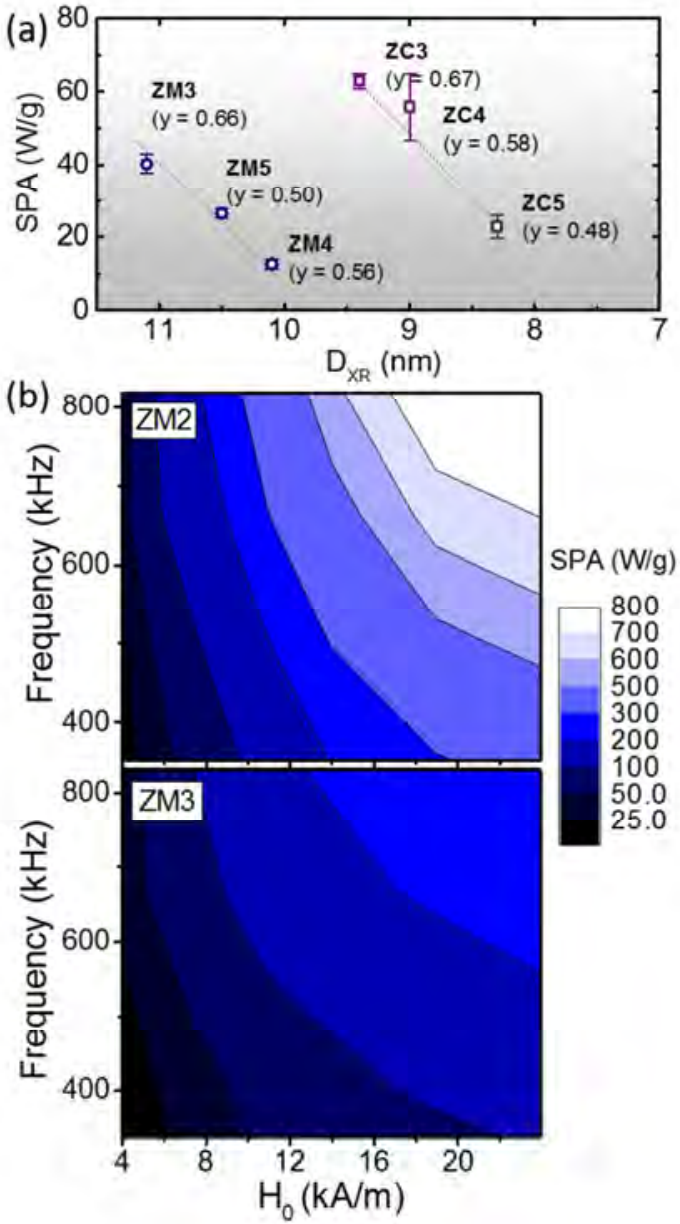

Figure 7. (a) Comparison between SPA values (at $12 \mathrm{kA} / \mathrm{m}$ and $265 \mathrm{kHz}$ ) of some $\mathrm{Zn}-\mathrm{Mn}$ and Zn-Co based ferrites NPs with similar Mn or Co fractions (y) and (b) SPA results obtained ad different frequencies and magnetic field amplitudes.

Figure $7 \mathrm{~b}$ illustrates the SPA values at different frequencies $(265-831 \mathrm{kHz})$ and field amplitudes $(4-24 \mathrm{kA} / \mathrm{m})$ for NPs of ZM2 and ZM3 samples, both dispersed in water. At $24 \mathrm{kA} / \mathrm{m}$ and $831 \mathrm{kHz}$, we obtain a SPA value of 799 $\mathrm{W} / \mathrm{g}$ for ZM2 sample decreasing to $304 \mathrm{~W} / \mathrm{g}$ for ZM3 one. Even if the $H_{0} f$ product obtained with the field amplitude and frequency of our experiments is well above the human tolerable limitation ${ }^{86}$ of $H_{0} f<4.85 \times 10^{8} \mathrm{Am}^{-1} \mathrm{~s}^{-1}$, these SPA values are comparable with others reported in similar or even more severe conditions. In such magnetically soft ferrites, increasing both NPs' diameter and saturation magnetization (by slightly modifying the synthesis conditions) might be the key to increase the SPA. However, the size should not overcome the limit where Brownian relaxation becomes the dominant process since the heating efficiency of the NPs must be maintained in medium with larger viscosities. Nonetheless, considering that the NPs' diameters of some approved MRI contrast agents lies in the range of $20-150 \mathrm{~nm}{ }^{87}$ and that the size reduction 
leads to larger longitudinal relaxivities ${ }^{88,89}$, our rather small NPs could be also suitable as MRI contrast agents in a theranostic approach.

The SPAs obtained for Zn-Co series are very similar when compared to those of Zn-Mn ones, except for ZM2 NPs, which exhibits the highest value, most likely related to its higher diameter and saturation magnetization. In fact, considering that $\mathrm{Zn}$-Co series of samples present the highest anisotropy contrast between core and shell materials, a larger exchange coupling at the interface would be expected, which could increase heating efficiency ${ }^{41,44-46}$. Here, one would wonder about the nature of this exchange coupling, an issue which is not very clear in the literature. Indeed, an exchange bias field has already been observed in ultrasmall core/shell ferrite NPs $(\sim 3 \mathrm{~nm})$, synthesized by the same chemical route as used here ${ }^{48,63}$. The exchange bias field originates at an internal interface between the well-ordered ferrimagnetic core and a surface layer of disordered spins ${ }^{72}$. This exchange bias is found much larger for NPs based on harder ferrite core ${ }^{48}$. Nevertheless, this interfacial exchange coupling typically vanishes at temperatures above $50 \mathrm{~K}^{48}$, and has not been observed with the present NPs at room temperature. Thus, in our case, it seems highly improbable that this exchange bias field can be involved in the mechanism responsible for increasing the SPA. However, the thickness of the chemical shell of the here probed NPs is $\sim 0.5 \mathrm{~nm}^{59,73}$, which is smaller than their magnetically disordered surface layer. Then, the elaboration and study of core/shell NPs with different proportions of core and shell ferrites phases may enlighten the nature of the exchange coupling and its contribution to the heating efficiency in hyperthermia.

\section{CONCLUSIONS}

$\mathrm{Zn}_{\mathrm{x}} \mathrm{M}_{\mathrm{y}} \mathrm{Fe}_{\mathrm{z}} \mathrm{O}_{4}(\mathrm{M}=\mathrm{Mn}, \mathrm{Co}$ ) NPs were synthesized by hydrothermal coprecipitation. Zn substitution yielded deviations from nominal stoichiometry in both sets of samples and a significant change in diameter, more pronounced in the $\mathrm{Zn}$-Mn series. We succeeded in producing a core/shell structure, consisting of a mixed ferrite core coated with a thin layer of maghemite. This strategy allowed to disperse the $\mathrm{Zn}_{\mathrm{x}} \mathrm{M}_{\mathrm{y}} \mathrm{Fe}_{\mathrm{z}} \mathrm{O}_{4} @ \mathrm{y}-\mathrm{Fe}_{2} \mathrm{O}_{3}$ NPs in acidic medium to obtain long term stable ferrofluids.

Regarding the magnetic measurements properties of the NPs, we found that Zn substitution modulates the NPs' magnetization and anisotropy in different ways. The magnetic anisotropy of Zn-Co ferrite based NPs changes drastically from $K_{e f}=2.5 \times 10^{5} \mathrm{~J} / \mathrm{m}^{3}$ (low zinc content) to $5.3 \times 10^{4} \mathrm{~J} / \mathrm{m}^{3}$ (high zinc content), while anisotropy of $\mathrm{Zn}-\mathrm{Mn}$ ferrite based NPs changed less than $40 \%$ $\left(0.9-1.4 \times 10^{4} \mathrm{~J} / \mathrm{m}^{3}\right)$ for any $\mathrm{Zn}$ contents.

Consistently, these differences of magnetic anisotropies were reflected in the behavior of the NPs' thermomagnetic coefficient as a function of their Zn content (investigated in pressed powders). Tc values decreased with the Zn content for both series of samples resulting in NPs with lower Tc values than other iron oxides, specially magnetite and maghemite. Decreasing Tc down to hyperthermic tempera- tures would be very interesting to avoid overheating in biological media. In addition to the magnetization decrease, augmenting the thermal energy allied to the thermally-induced changes to both viscosity and magnetic anisotropy reflect in a decrease of $\tau$, thus shifting the peak of absorption to higher NP diameters possibly hindering even further the heating efficiency.

The two series of NPs having either hard or soft ferrite cores and soft ferrite shell, did not present evidences of any interfacial exchange coupling contribution to their power absorption efficiency, as previous reports suggested. Thus, in the absence of exchange coupling effects, synthesizing NPs based on Zn-Mn ferrites with both larger sizes and $\mathrm{Zn}$ content, would be the key to increase power absorption efficiency while achieving a self-regulated hyperthermia.

In a very near future, we intend to improve the synthesis procedure in order to reach larger nanoparticle diameters and low polydispersity. Different strategies of synthesis routes and magnetic size sorting (using an ionic strengthinduced process as in Ref. ${ }^{79}$ ) would be one of the primary directions to be investigated.

\section{ASSOCIATED CONTENT}

\section{Supporting Information.}

Figure S1: X-ray diffractograms of core/shell NPs; Figure S2: lattice parameters of Zn-Mn and Zn-Co sets of samples; Figure S3: core/shell chemical structure of ZC3 sample; Figure S4: Curie temperature determination; Figure S5: hysteresis cycles at $300 \mathrm{~K}$ for NPs samples in powder form; Table S1: chemical characterization of core/shell NPs; Table S2: structural characteristics of core/shell NPs; Table S3: magnetic properties of the samples.

\section{AUTHOR INFORMATION}

\author{
Corresponding Author \\ *E-mail: depeyrot@fis.unb.br. Phone: +55 (61)3107-7781
}

Notes

The authors declare no competing financial interest.

\section{ACKNOWLEDGMENT}

This work was supported by CNPq, CAPES and FAP/DF brazilian agencies. R. Cabreira Gomes is very grateful for his CNPq grant, contract 202340/2015-5. We thank Laboratório Nacional de Luz Síncrotron (LNLS) for XRPD measurements on D12A-XRD1 beamline and LMA (Laboratorio de Microscopías Avanzadas - University of Zaragoza) for STEM measurements. Partial support from MINECO, Spain, is acknowledged. Special thanks to Dr. Beatriz Sanz for technical assistance on hyperthermia measurements and Dr. Fernando H. Martins for XRPD measurements.

\section{REFERENCES}

(1) Jordan, A.; Wust, P. Inductive Heating of Ferrimagnetic Particles and Magnetic Fluids: Physical Evaluation of Their Potential for Hyperthermia. Int. J. Hyperth. 1993, 9, 51-68. 
Wlodarczyk, W.; Sander, B.; Vogl, T.; Felix, R. Effects of Magnetic Fluid Hyperthermia (MFH) on C3H Mammary Carcinoma in Vivo. Int. J. Hyperth. 1997, 13, 587-605.

(3) Goya, G. F.; Grazú, V.; Ibarra, M. R. Magnetic Nanoparticles for Cancer Therapy. Curr. Nanosci. 2008, 4, 1-16.

(4) Pankhurst, Q. a; Thanh, N. T. K.; Jones, S. K.; Dobson, J. Progress in Applications of Magnetic Nanoparticles in Biomedicine. J. Phys. D. Appl. Phys. 2009, 42, 224001224016.

(5) Rosensweig, R. E. Heating Magnetic Fluid with Alternating Magnetic Field. J. Magn. Magn. Mater. 2002, 252, 370-374.

(6) Carrey, J.; Mehdaoui, B.; Respaud, M. Simple Models for Dynamic Hysteresis Loop Calculations of Magnetic SingleDomain Nanoparticles: Application to Magnetic Hyperthermia Optimization. J. Appl. Phys. 2011, 109, 083921-083938.

(7) Néel, L. Propriétées Magnétiques Des Ferrites; Férrimagnétisme et Antiferromagnétisme. Ann. Phys. 1948, 3, 137-198.

(8) Fannin, P. C.; Charles, S. W. On the Calculation of the Néel Relaxation Time in Uniaxial Single-Domain Ferromagnetic Particles. J. Phys. D. Appl. Phys. 1994, 27, 185-188.

(9) Usov, N. A. Low Frequency Hysteresis Loops of Superparamagnetic Nanoparticles with Uniaxial Anisotropy Low Frequency Hysteresis Loops of Superparamagnetic Nanoparticles with Uniaxial Anisotropy. J. Appl. Phys. 2010, 107, 123909-123921.

(10) Périgo, E. A.; Hemery, G.; Sandre, O.; Ortega, D.; Garaio, E.; Plazaola, F.; Teran, F. J. Fundamentals and Advances in Magnetic Hyperthermia. Appl. Phys. Rev. 2015, 2, 4130241337.

(11) Fortin, J. P.; Wilhelm, C.; Servais, J.; Ménager, C.; Bacri, J. C.; Gazeau, F. Size-Sorted Anionic Iron Oxide Nanomagnets as Colloidal Mediators for Magnetic Hyperthermia. J. Am. Chem. Soc. 2007, 129, 2628-2635.

(12) De La Presa, P.; Luengo, Y.; Multigner, M.; Costo, R.; Morales, M. P.; Rivero, G.; Hernando, A. Study of Heating Efficiency as a Function of Concentration, Size, and Applied Field in GFe203 Nanoparticles. J. Phys. Chem. C 2012, 116, 2560225610.

(13) Lima, E.; Torres, T. E.; Rossi, L. M.; Rechenberg, H. R.; Berquo, T. S.; Ibarra, A.; Marquina, C.; Ibarra, M. R.; Goya, G. F. Size Dependence of the Magnetic Relaxation and Specific Power Absorption in Iron Oxide Nanoparticles. J. Nanoparticle Res. 2013, 15, 1654-1665.

(14) Nemati, Z.; Alonso, J.; Martinez, L. M.; Khurshid, H.; Garaio, E.; Garcia, J. A.; Phan, M. H.; Srikanth, H. Enhanced Magnetic Hyperthermia in Iron Oxide Nano-Octopods: Size and Anisotropy Effects. J. Phys. Chem. C 2016, 120, 8370-8379.

(15) Mehdaoui, B.; Meffre, A.; Carrey, J.; Lachaize, S.; Lacroix, L. M.; Gougeon, M.; Chaudret, B.; Respaud, M. Optimal Size of Nanoparticles for Magnetic Hyperthermia: A Combined Theoretical and Experimental Study. Adv. Funct. Mater. 2011, 21, 4573-4581.

(16) Goya, G. F.; Lima, E.; Arelaro, A. D.; Torres, T.; Rechenberg, H. R.; Rossi, L.; Marquina, C.; Ibarra, M. R.; Paulo, I. D. F. D. S. Paulo, S.; et al. Magnetic Hyperthermia With Fe304 Nanoparticles: The Influence of Particle Size on Energy Absorption. IEEE Trans. Magn. 2008, 44, 4444-4447.

(17) Vallejo-Fernandez, G.; Whear, O.; Roca, A. G.; Hussain, S.; Timmis, J.; Patel, V.; O'Grady, K. Mechanisms of Hyperthermia in Magnetic Nanoparticles. J. Phys. D. Appl. Phys. 2013, 46, 312001-312007.

(18) Lartigue, L.; Hugounenq, P.; Alloyeau, D.; Clarke, S. P.; Le, M.; Bazzi, R.; Brougham, D. F.; Wilhelm, C.; Gazeau, F. Cooperative Organization in Iron Oxide Multi-Core Nanoparticles Potentiates Their Efficiency as Heating Mediators and MRI Contrast Agents. ACS Nano 2012, 6 , 10935-10949.

(19) Noh, S. H.; Na, W.; Jang, J. T.; Lee, J. H.; Lee, E. J.; Moon, S. H.; Lim, Y.; Shin, J. S.; Cheon, J. Nanoscale Magnetism Control via
Surface and Exchange Anisotropy for Optimized Ferrimagnetic Hysteresis. Nano Lett. 2012, 12, 3716-3721.

(20) Guardia, P.; Di Corato, R.; Lartigue, L.; Wilhelm, C.; Espinosa A.; Garcia-Hernandez, M.; Gazeau, F.; Manna, L.; Pellegrino, T. Water-Soluble Iron Oxide Nanocubes with High Values of Specific Absorption Rate for Cancer Cell Hyperthermia Treatment. ACS Nano 2012, 6, 3080-3091.

(21) Martinez-Boubeta, C.; Simeonidis, K.; Makridis, A.; Angelakeris, M.; Iglesias, O.; Guardia, P.; Cabot, A.; Yedra, L.; Estradé, S.; Peiró, F.; et al. Learning from Nature to Improve the Heat Generation of Iron-Oxide Nanoparticles for Magnetic Hyperthermia Applications. Sci. Rep. 2013, 3, 1652-1660.

(22) Das, R.; Alonso, J.; Nemati Porshokouh, Z.; Kalappattil, V.; Torres, D.; Phan, M. H.; Garaio, E.; García, J. Á.; Sanchez Llamazares, J. L.; Srikanth, H. Tunable High Aspect Ratio Iron Oxide Nanorods for Enhanced Hyperthermia. J. Phys. Chem. C 2016, 120, 10086-10093.

(23) Lima, E.; De Biasi, E.; Zysler, R. D.; Vasquez Mansilla, M. Mojica-Pisciotti, M. L.; Torres, T. E.; Calatayud, M. P.; Marquina, C.; Ricardo Ibarra, M.; Goya, G. F. Relaxation Time Diagram for Identifying Heat Generation Mechanisms in Magnetic Fluid Hyperthermia. J. Nanoparticle Res. 2014, 16, 2791-2802.

(24) Fantechi, E.; Innocenti, C.; Albino, M.; Lottini, E.; Sangregorio C. Influence of Cobalt Doping on the Hyperthermic Efficiency of Magnetite Nanoparticles. J. Magn. Magn. Mater. 2015, 380, 365-371.

(25) Mameli, V.; Musinu, A.; Ardu, A.; Ennas, G.; Peddis, D. Niznansky, D.; Sangregorio, C.; Innocenti, C.; Thanh, N. T. K.; Cannas, C. Studying the Effect of Zn-Substitution on the Magnetic and Hyperthermic Properties of Cobalt Ferrite Nanoparticles. Nanoscale 2016, 8 (19), 10124-10137.

(26) Suto, M.; Hirota, Y.; Mamiya, H.; Fujita, A.; Kasuya, R.; Tohji, K.; Jeyadevan, B. Heat Dissipation Mechanism of Magnetite Nanoparticles in Magnetic Fluid Hyperthermia. J. Magn. Magn. Mater. 2009, 321, 1493-1496.

(27) Coral, D. F.; Zélis, P. M.; Marciello, M.; Puerto, M. Del; Craievich, A.; Sanchez, F. H.; Raap, M. B. F. Van. On the Effect of Nanoclustering and Dipolar Interactions in Heat Generation for Magnetic. Langmuir 2016, 32, 1201-1213.

(28) Dennis, C. L.; Jackson, a J.; Borchers, J. a; Hoopes, P. J.; Strawbridge, R.; Foreman, a R.; van Lierop, J.; Grüttner, C.; Ivkov, R. Nearly Complete Regression of Tumors via Collective Behavior of Magnetic Nanoparticles in Hyperthermia. Nanotechnology 2009, 20, 395103-395110.

(29) Serantes, D.; Baldomir, D.; Simeonidis, K.; Angelakeris, M.; Natividad, E.; Castro, M.; Chen, D.; Sanchez, A.; Balcells, L. I.; Martínez, B.; et al. Influence of Dipolar Interactions on Hyperthermia Properties of Ferromagnetic Particles Influence of Dipolar Interactions on Hyperthermia Properties of Ferromagnetic Particles. J. Appl. Phys. 2010, 108, 073918-073923.

(30) Urtizberea, A.; Natividad, E.; Arizaga, A.; Castro, M.; Mediano, A. Specific Absorption Rates and Magnetic Properties of Ferrofluids with Interaction Effects at Low Concentrations. J. Phys. Chem. C 2010, 114, 4916-4922.

(31) Branquinho, L. C.; Carrião, M. S.; Costa, A. S.; Zufelato, N.; Sousa, M. H.; Miotto, R.; Ivkov, R.; Bakuzis, A. F. Effect of Magnetic Dipolar Interactions on Nanoparticle Heating Efficiency: Implications for Cancer Hyperthermia. Sci. Rep. 2013, 3, 02887-02897.

(32) Sadat, M. E.; Patel, R.; Sookoor, J.; Bud'Ko, S. L.; Ewing, R. C.; Zhang, J.; Xu, H.; Wang, Y.; Pauletti, G. M.; Mast, D. B.; et al. Effect of Spatial Confinement on Magnetic Hyperthermia via Dipolar Interactions in Fe304 Nanoparticles for Biomedical Applications. Mater. Sci. Eng. C 2014, 42, 52-63.

(33) Martinez-Boubeta, C.; Simeonidis, K.; Serantes, D.; CondeLeborán, I.; Kazakis, I.; Stefanou, G.; Peña, L.; Galceran, R.; Balcells, L.; Monty, C.; et al. Adjustable Hyperthermia Response of Self-Assembled Ferromagnetic Fe-MgO Core- 
Shell Nanoparticles by Tuning Dipole-Dipole Interactions. Adv. Funct. Mater. 2012, 22, 3737-3744.

Saville, S. L.; Qi, B.; Baker, J.; Stone, R.; Camley, R. E.; Livesey, K. L.; Ye, L.; Crawford, T. M.; Thompson Mefford, O. The Formation of Linear Aggregates in Magnetic Hyperthermia: Implications on Specific Absorption Rate and Magnetic Anisotropy. J. Colloid Interface Sci. 2014, 424, 141-151.

(35) Serantes, D.; Simeonidis, K.; Angelakeris, M.; Chubykalofesenko, O.; Marciello, M.; Morales, P.; Baldomir, D.; Martinez-boubeta, C. Multiplying Magnetic Hyperthermia Response by Nanoparticle Assembling. J. Phys. Chem. C 2014, 118, 5927-5934.

(36) Guibert, C.; Dupuis, V.; Peyre, V.; Fresnais, J. Hyperthermia of Magnetic Nanoparticles: Experimental Study of the Role of Aggregation. J. Phys. Chem. C 2015, 119, 28148-28154.

(37) Myrovali, E.; Maniotis, N.; Makridis, A.; Terzopoulou, A.; Ntomprougkidis, V.; Simeonidis, K.; Sakellari, D.; Kalogirou, O.; Samaras, T.; Salikhov, R.; et al. Arrangement at the Nanoscale: Effect on Magnetic Particle Hyperthermia. Sci. Rep. 2016, 6, 37934-37945.

(38) Hemery, G.; Keyes, A. C.; Garaio, E.; Rodrigo, I.; Garcia, J. A.; Plazaola, F.; Garanger, E.; Sandre, O. Tuning Sizes, Morphologies, and Magnetic Properties of Mono-vs. MultiCore Iron Oxide Nanoparticles through Control of Added Water in the Polyol Synthesis. Inorg. Chem. 2017, 56, 82328243.

(39) Habib, A. H.; Ondeck, C. L.; Chaudhary, P.; Bockstaller, M. R. McHenry, M. E. Evaluation of Iron-Cobalt/ferrite Core-Shell Nanoparticles for Cancer Thermotherapy. J. Appl. Phys. 2008, 103, 07A307-07A310.

(40) Mamiya, H. Recent Advances in Understanding Magnetic Nanoparticles in AC Magnetic Fields and Optimal Design for Targeted Hyperthermia. J. Nanomater. 2013, 2013, 752973752990.

(41) López-Ortega, A.; Estrader, M.; Salazar-Alvarez, G.; Roca, A. G.; Nogués, J. Applications of Exchange Coupled Bi-Magnetic Hard/soft and Soft/hard Magnetic Core/shell Nanoparticles. Phys. Rep. 2015, 553, 1-94.

(42) Obaidat, I.; Issa, B.; Haik, Y. Magnetic Properties of Magnetic Nanoparticles for Efficient Hyperthermia. Nanomaterials 2015, 5, 63-89.

(43) Liu, F.; Hou, Y.; Gao, S. Exchange-Coupled Nanocomposites: Chemical Synthesis, Characterization and Applications. Chem. Soc. Rev. 2014, 43, 8098-8113.

(44) Lee, J.-H.; Jang, J.-T.; Choi, J.-S.; Moon, S. H.; Noh, S.-H.; Kim, J.W.; Kim, J.-G.; Kim, I.-S.; Park, K. I.; Cheon, J. ExchangeCoupled Magnetic Nanoparticles for Efficient Heat Induction. Nat. Nanotechnol. 2011, 6, 418-422.

(45) Liebana-Viñas, S.; Simeonidis, K.; Wiedwald, U.; Li, Z.-A.; Myrovali, E.; Makridis, A.; Sakellari, D.; Vourlias, G.; Spasova, M.; Angelakeris, M. Optimum Nanoscale Design in Ferrite Based Nanoparticles for Magnetic Particle Hyperthermia. RSC Adv. 2016, 6, 72918-72925.

(46) Carrião, M. S.; Bakuzis, A. F. Mean-Field and Linear Regime Approach to Magnetic Hyperthermia of Core-shell Nanoparticles: Can Tiny Nanostructures Fight Cancer? Nanoscale 2016, 8, 8363-8377.

(47) Vasilakaki, M.; Binns, C.; Trohidou, K. N. Susceptibility Losses in Heating of Magnetic Core/shell Nanoparticles for Hyperthermia: A Monte Carlo Study of Shape and Size Effects. Nanoscale 2015, 7, 7753-7762.

(48) Cabreira-Gomes, R.; G. Silva, F.; Aquino, R.; Bonville, P.; Tourinho, F. A.; Perzynski, R.; Depeyrot, J. Exchange Bias of $\mathrm{MnFe} 204 @ \gamma-\mathrm{Fe} 2 \mathrm{O} 3$ and CoFe2O4@y-Fe2O3 Core/shell Nanoparticles. J. Magn. Magn. Mater. 2014, 368, 409-414.

(49) Arulmurugan, R.; Jeyadevan, B.; Vaidyanathan, G.; Sendhilnathan, S. Effect of Zinc Substitution on Co-Zn and Mn-Zn Ferrite Nanoparticles Prepared by Co-Precipitation. J. Magn. Magn. Mater. 2005, 288, 470-477.

(50) Arulmurugan, R.; Vaidyanathan, G.; Sendhilnathan, S.; Jeyadevan, B. Mn-Zn Ferrite Nanoparticles for Ferrofluid
Preparation: Study on Thermal-magnetic Properties. Magn. Magn. Mater. 2006, 298, 83-94.

(51) Rath, C.; Sahu, K. K.; Anand, S.; Date, S. K.; Mishra, N. C.; Das, R. P. Preparation and Characterization of Nanosize Mn-Zn Ferrite. J. Magn. Magn. Mater. 1999, 202, 77-84.

(52) Xuan, Y.; Li, Q.; Yang, G. Synthesis and Magnetic Properties of Mn-Zn Ferrite Nanoparticles. J. Magn. Magn. Mater. 2007, 312, 464-469.

(53) Gopalan, E. V.; Al-Omari, I. a.; Malini, K. a.; Joy, P. a.; Sakthi Kumar, D.; Yoshida, Y.; Anantharaman, M. R. Impact of Zinc Substitution on the Structural and Magnetic Properties of Chemically Derived Nanosized Manganese Zinc Mixed Ferrites. J. Magn. Magn. Mater. 2009, 321, 1092-1099.

(54) Hanini, A.; Lartigue, L.; Gavard, J.; Kacem, K.; Wilhelm, C.; Gazeau, F.; Chau, F.; Ammar, S. Journal of Magnetism and Magnetic Materials Zinc Substituted Ferrite Nanoparticles with $\mathrm{Zn0.9Fe2.104} \mathrm{Formula} \mathrm{Used} \mathrm{as} \mathrm{Heating} \mathrm{Agents} \mathrm{for} \mathrm{in}$ Vitro Hyperthermia Assay on Glioma Cells. J. Magn. Magn. Mater. 2016, 416, 315-320.

(55) Lin, M.; Zhang, D.; Huang, J.; Zhang, J.; Xiao, W.; Yu, H.; Zhang, L.; Ye, J. The Anti-Hepatoma Effect of Nanosized Mn-Zn Ferrite Magnetic Fluid Hyperthermia Associated with Radiation in Vitro and in Vivo. Nanotechnology 2013, 24, 255101-255109.

(56) Lin, M.; Huang, J.; Sha, M. Recent Advances in Nanosized MnZn Ferrite Magnetic Fluid Hyperthermia for Cancer Treatment. J. Nanosci. Nanotechnol. 2014, 14, 792-802.

(57) Tourinho, F. A.; Franck, R.; Massart, R. Aqueous Ferrofluids Based on Manganese and Cobalt Ferrites. J. Mater. Sci. 1990, 25, 3249-3254.

(58) Sousa, M. H.; Tourinho, F. A.; Jose, G.; Lara, M. C. F. L. New Electric Double-Layered Magnetic Fluids Based on Copper Nickel , and Zinc Ferrite Nanostructures. J. Phys. Chem. B 2001, 105, 1168-1175.

(59) Gomes, J. D. A.; Sousa, M. H.; Tourinho, F. A.; Aquino, R.; Silva, G. J.; Depeyrot, J.; Dubois, E.; Perzynski, R. Synthesis of Core Shell Ferrite Nanoparticles for Ferrofluids: Chemical and Magnetic Analysis. J. Phys. Chem. C 2008, 112, 6220-6227.

(60) Campos, A. F. C.; Tourinho, F. A.; Da Silva, G. J.; Lara, M. C. F. L.; Depeyrot, J. Nanoparticles Superficial Density of Charge in Electric Double-Layered Magnetic Fluid: A Conductimetric and Potentiometric Approach. Eur. Phys. J. E 2001, 6, 29-35.

(61) Campos, A. F. C.; Aquino, R.; Tourinho, F. A.; Paula, F. L. O.; Depeyrot, J. Influence of the Spatial Confinement at Nanoscale on the Structural Surface Charging in Magnetic Nanocolloids. Eur. Phys. J. E. Soft Matter 2013, 36, 42-53.

(62) Alves, C. R.; Aquino, R.; Depeyrot, J.; Tourinho, F. a.; Dubois, E.; Perzynski, R. Superparamagnetic Relaxation Evidences Large Surface Contribution for the Magnetic Anisotropy of MnFe204 Nanoparticles of Ferrofluids. J. Mater. Sci. 2007, 42, 2297-2303.

(63) Silva, F. G.; Aquino, R.; Tourinho, F. A.; Stepanov, V. I.; Raikher, Y. L.; Perzynski, R.; Depeyrot, J. The Role of Magnetic Interactions in Exchange Bias Properties of MnFe204@y-Fe203 Core/shell. J. Phys. D. Appl. Phys. 2013, 46, 285003-285012.

(64) Martins, F. H.; Silva, F. G.; Paula, F. L. 0.; Gomes, J. D. A Aquino, R.; Mestnik-filho, J.; Bonville, P.; Porcher, F. Local Structure of Core-Shell MnFe204 $+\delta$-Based Nanocrystals: Cation Distribution and Valence States of Manganese Ions. J. Phys. Chem. C 2017, 121, 8982-8991.

(65) Auzans, E.; Zins, D.; Blums, E.; Massart, R. Synthesis and Properties of Mn-Zn Ferrite Ferrofluids. J. Mater. Sci. 1999, $34,1253-1260$.

(66) Wang, J.; Zeng, C.; Peng, Z.; Chen, Q. Synthesis and Magnetic Properties of $\mathrm{Zn} 1-\mathrm{xMnxFe} 204$ Nanoparticles. Phys. B Condens. Matter 2004, 349, 124-128.

(67) Upadhyay, C.; Verma, H. C.; Rath, C.; Sahu, K. K.; Anand, S.; Das, R. P.; Mishra, N. C. Mossbauer Studies of Nanosize Mn1xZnxFe204. J. Alloys Compd. 2001, 326, 94-97.

(68) Rath, C.; Anand, S.; Das, R. P.; Sahu, K. K.; Kulkarni, S. D. 
Dependence on Cation Distribution of Particle Size, Lattice Parameter, and Magnetic Properties in Nanosize $\mathrm{Mn}-\mathrm{Zn}$ Ferrite. J. Appl. Phys. 2002, 91, 2211-2215.

(69) Beji, Z.; Sun, M.; Smiri, L. S.; Herbst, F.; Mangeney, C.; Ammar S. Polyol Synthesis of Non-Stoichiometric Mn-Zn Ferrite Nanocrystals: Structural /microstructural Characterization and Catalytic Application. RSC Adv. 2015, 5, 65010-65022.

(70) Vamvakidis, K.; Katsikini, M.; Sakellari, D.; Paloura, E. C.; Kalogirou, O.; Dendrinou-Samara, C. Reducing the Inversion Degree of MnFe204 Nanoparticles through Synthesis to Enhance Magnetization: Evaluation of Their (1)H NMR Relaxation and Heating Efficiency. Dalt. Trans. 2014, 43, 12754-12765.

(71) Gomes, J. A.; Azevedo, G. M.; Depeyrot, J.; Mestnik-Filho, J.; Paula, F. L. 0.; Tourinho, F. A.; Perzynski, R. Structural, Chemical, and Magnetic Investigations of Core-Shell Zinc Ferrite Nanoparticles. J. Phys. Chem. C 2012, 116, 2428124291.

(72) Aquino, R.; Depeyrot, J.; Sousa, M.; Tourinho, F.; Dubois, E.; Perzynski, R. Magnetization Temperature Dependence and Freezing of Surface Spins in Magnetic Fluids Based on Ferrite Nanoparticles. Phys. Rev. B 2005, 72, 184435184445.

(73) Sousa, E. C.; Rechenberg, H. R.; Depeyrot, J.; Gomes, J. A.; Aquino, R.; Tourinho, F. A.; Dupuis, V.; Perzynski, R. In-Field Mossbauer Study of Disordered Surface Spins in Core/shell Ferrite Nanoparticles. J. Appl. Phys. 2009, 106, 093901093908.

(74) Stoner, E. C.; Wohlfarth, E. P. A Mechanism of Magnetic Hysteresis in Heterogeneous Alloys. Philosophical Transactions of the Royal Society A: Mathematical, Physical and Engineering Sciences. 1948, pp 599-642.

(75) Cullity, B. D.; Graham, C. D. Introduction to Magnetic Materials, 2nd Ed.; IEE Press, Wiley, 2009.

(76) Jnaneshwara, D. M.; Avadhani, D. N.; Daruka Prasad, B.; Nagabhushana, B. M.; Nagabhushana, H.; Sharma, S. C.; Prashantha, S. C.; Shivakumara, C. Effect of Zinc Substitution on the Nanocobalt Ferrite Powders for Nanoelectronic Devices. J. Alloys Compd. 2014, 587, 50-58.

(77) de Biasi, R. S. De; Cardoso, L. H. G. A Simple Model for the Magnetocrystalline Anisotropy in Mixed Ferrite Nanoparticles. Phys. B Phys. Condens. Matter 2012, 407, 3893-3896.

(78) Rondinone, A. J.; Liu, C.; John Zhang, Z. Determination of Magnetic Anisotropy Distribution and Anisotropy Constant of Manganese Spinel Ferrite Nanoparticles. J. Phys. Chem. B 2001, 105, 7967-7971.

(79) Gazeau, F.; Bacri, J. C.; Gendron, F.; Perzynski, R.; Raikher, Y. L.; Stepanov, V. I.; Dubois, E. Magnetic Resonance of Ferrite Nanoparticles: Evidence of Surface Effects. J. Magn. Magn. Mater. 1998, 186, 175-187.

(80) Andersson, M. S.; Mathieu, R.; Lee, S. S.; Normile, P. S.; Singh, G.; Nordblad, P.; De Toro, J. A. Size-Dependent Surface Effects in Maghemite Nanoparticles and Its Impact on Interparticle Interactions in Dense Assemblies. Nanotechnology 2015, 26, 475703-475709.

(81) Beji, Z.; Hanini, A.; Smiri, L. S.; Gavard, J.; Kacem, K.; Villain F.; Gren, J. Magnetic Properties of Zn-Substituted MnFe204 Nanoparticles Synthesized in Polyol as Potential Heating Agents for Hyperthermia . Evaluation of Their Toxicity on Endothelial Cells. Chem. Mater. 2010, 22, 5420-5429.

(82) Cornell, R. M.; Schwertmann, U. The Iron Oxides, 2nd Ed.; WILEY - VCH, 2003.

(83) Arulmurugan, R.; Vaidyanathan, G.; Sendhilnathan, S.; Jeyadevan, B. Thermomagnetic Properties of Co1xZnxFe204 (x=0.1-0.5) Nanoparticles. J. Magn. Magn. Mater. 2006, 303, 131-137.

(84) Garaio, E.; Sandre, O.; Collantes, J.-M.; Garcia, J. A.; Mornet, S.; Plazaola, F. Specific Absorption Rate Dependence on Temperature in Magnetic Field Hyperthermia Measured by Dynamic Hysteresis Losses (Ac Magnetometry).
(85) Giri, J.; Pradhan, P.; Sriharsha, T.; Bahadur, D. Preparation and Investigation of Potentiality of Different Soft Ferrites for Hyperthermia Applications. J. Appl. Phys. 2005, 97, 10 Q916.

(86) Atkinson, W. J.; Brezovich, I. A.; Chakraborty, D. P. Usable Frequencies in Hyperthermia with Thermal Seeds. IEEE Trans. Biomed. Eng. 1984, BME-31, 70-75.

(87) Wang, Y. X. J.; Hussain, S.; Krestin, G. Superparamagnetic Iron Oxide Contrast Agents : Physicochemical Characteristics and Applications in MR Imaging. Eur. Radiol. 2001, 11, 23192331.

(88) Tromsdorf, U. I.; Bigall, N. C.; Kaul, M. G.; Bruns, O. T.; Nikolic, M. S.; Mollwitz, B.; Sperling, R. a; Reimer, R.; Hohenberg, H.; Parak, W. J.; et al. Size and Surface Effects on the MRI Relaxivity of Manganese Ferrite Nanoparticle Contrast Agents. Nano Lett. 2007, 7, 2422-2427.

(89) Li, Z.; Wang, S. X.; Sun, Q.; Zhao, H. L.; Lei, H.; Lan, M. B. Cheng, Z. X.; Wang, X. L.; Dou, S. X.; Qing, G.; et al. Ultrasmall Manganese Ferrite Nanoparticles as Positive Contrast Agent for Magnetic Resonance Imaging. Adv. Healthc. Mater. 2013, 2, 958-964. 
TOC Graphic:

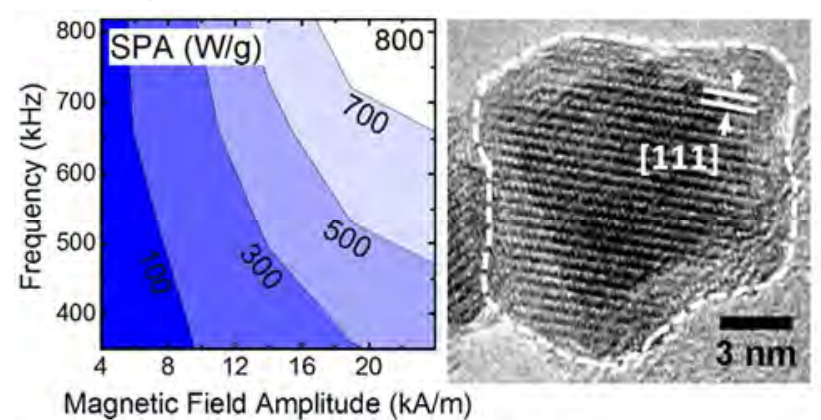

$13 \quad$ Magnetic Field Amplitude $(\mathrm{kA} / \mathrm{m})$ 